\title{
Control systems design based on classical dynamics
}

\author{
Wang Funing ${ }^{1}$, Kai Pingan ${ }^{2}$ \\ ${ }^{1}$ Ningdong Power Plant, Gouhua Power Group, Ningdong town, Ningxia, P. R. C. \\ ${ }^{2}$ Energy Research Institute, Development and Reformation Committee of State, Beijing, P. R. C.
}

\section{Email address:}

wangfn7119@163.com (Wang Funing),pingank@aliyun.com (Kai Pingan)

\section{To cite this article:}

Wang Funing, Kai Pingan. Control Systems Design Based on Classical Dynamics. Automation, Control and Intelligent Systems. Vol. 2, No. 5, 2014, pp. 81-86. doi: 10.11648/j.acis.20140205.12

\begin{abstract}
The paper presents unity between control systems and classical dynamics. A state observer is constructed based on uniformly accelerated motion. It is known $\mathrm{F}=\mathrm{ma}$ in Newtonian motion equation is considered as a control input force which functions on the controlled plant process. The designed control system is of good robust performance.
\end{abstract}

Keywords: Uniformly Accelerated Motion, Newtonian Mechanics, State Observer, Kalman Filter, Robust Performance

\section{Introduction}

Design control systems in modern control theory always needs a mathematics model of controlled plant, however the model is not exactly obtained and the model is uncertain in general, these limits led to the poor control system performance.

The paper designs a control system based on Classical dynamics without exact plant model, and the systems are of good robust performances.

\section{State Observer Based on Uniformly Accelerated Motion}

When a motion (/response) velocity of controlled plant process (/ body) is greatly less than velocity of light, we can describe its motion using uniformly acceleration motion:

$$
\begin{gathered}
S=S_{0}+V_{0} t+0.5 a t^{2} \\
\dot{S}=V=V_{0}+a t \\
\ddot{S}=\dot{V}=a
\end{gathered}
$$

where $S, V, a$ are respectively the position, velocity and acceleration of the body/process motion.

Assume a controlled second system with the random disturbance $v(t)$ :

$$
\ddot{y}=f(y, \dot{y}, v(t))+b u
$$

the system in state space is:

$$
\begin{gathered}
\dot{y}_{1}=y_{2} \\
\dot{y}_{2}=f\left(y_{1}, y_{2}, v(t)\right)+b u \\
y=y_{1}
\end{gathered}
$$

For a controlled plant process, assuming $z(1), z(2), \cdots, z(k)$, the $k$ measurement output data are obtained from the controlled plant output $y(k)$, sample(/control) period is $t_{s}$, the measurement equation is

$$
z(k)=y(k)+v(k)
$$

where: $E\left[v^{2}(k)\right]=r_{2}$

$$
E[v(k)]=0
$$

$v(k)$ is white noise, we can estimate the controlled plant output $y(k)$ using Exp. (1) based on $z(1), z(2), \cdots, z(k)$, when $t_{s}$ is very short,

$$
\begin{aligned}
& \hat{y}(k)=\hat{y}(k-1)+t_{s} \dot{\hat{y}}(k-1)+0.5 t_{s}^{2} \ddot{\hat{y}}(k-1) \\
& \dot{\hat{y}}(k)=\dot{\hat{y}}(k-1)+t_{s} \ddot{\hat{y}}(k-1) \\
& \ddot{\hat{y}}(k)=\ddot{\hat{y}}(k-1)
\end{aligned}
$$

where $\hat{y}(k), \dot{\hat{y}}(k)$ and $\ddot{\hat{y}}(k)$ are respectively the estimated values of $y(k), \dot{y}(k)$ and $\ddot{y}(k)$ at time $k$. 


$$
\text { let } \hat{Y}(k)=\left[\begin{array}{c}
\hat{y}(k) \\
\dot{\hat{y}}(k) \\
\ddot{\hat{y}}(k)
\end{array}\right] \text { and } \phi=\left[\begin{array}{ccc}
1 & t_{s} & t_{s}^{2} / 2 \\
0 & 1 & t_{s} \\
0 & 0 & 1
\end{array}\right]
$$

Exp. (5) can be written as:

$$
\hat{Y}(k)=\phi \hat{Y}(k-1)
$$

To improve estimate accuracy, the compensating for random disturbance is into acceleration estimate $\ddot{\hat{y}}(k)$ :

$$
\ddot{\hat{y}}(k)=\ddot{\hat{y}}(k-1)+w(k-1)
$$

where: $E[w(k)]=0, \quad E\left[w^{2}(k)\right]=r_{1}$

Exp.(7) becomes:

$$
\hat{Y}(k)=\phi \hat{Y}(k-1)+\Gamma w(k-1)
$$

where: $\quad \Gamma=\left[\begin{array}{lll}0 & 0 & 1\end{array}\right]^{T}$

measurement equation (4) is written as:

$$
z(k)=c \hat{Y}(k)+v(k)
$$

where: $c=\left[\begin{array}{lll}1 & 0 & 0\end{array}\right]$

Kalman filter theory can be used for state equation (9) and measurement equation(10), $\phi$ and $\Gamma$ are constant matrixes, meet $\operatorname{rank}(\phi, \Gamma)=n, \operatorname{rank}(\phi, c)=n$, so the system is completely controllable and observable in Kalman filter theory( the proof is given the following lemma), when $t_{s}$ is very short and filtering time is very long, the covariance matrix,

$$
\lim _{k \rightarrow \infty} P(k)=P,
$$

gain matrix

$$
\begin{gathered}
\operatorname{Lim}_{k \rightarrow \infty} K(k)=K, \\
K(k) \rightarrow K=[\alpha, \beta, \gamma]^{T} .
\end{gathered}
$$

We have the following time discrete observer to an unknown controlled plant:

$$
\left\{\begin{array}{l}
\hat{y}(k)=\hat{y}(k-1)+t_{s} \dot{\hat{y}}(k-1)+0.5 t_{t}^{2} \ddot{y}(k-1)+\alpha t_{s}(z(k)-\hat{y}(k-1)) \\
\hat{\hat{y}}(k)=\hat{\hat{y}}(k-1)+t_{s} \hat{\hat{y}}(k-1)+\beta t_{s}(z(k)-\hat{y}(k-1)) \\
\ddot{\hat{y}}(k)=\hat{y}(k-1)+\gamma_{s}(z(k)-\hat{y}(k-1))
\end{array}\right.
$$

$F=m a$ in the classical dynamics. Referencing Exp.(3), the control input $u$ is considered as a force which functions on the controlled plant, $u=F$, and $a=\theta u$, where $F$ is the force and $a$ is the acceleration.

As $\ddot{\hat{y}}(k)=(\dot{\hat{y}}(k)-\dot{\hat{y}}(k-1)) / t_{s}$ is an estimate to the acceleration of the system,

$$
\ddot{\hat{y}}(k)=(\dot{\hat{y}}(k)-\dot{\hat{y}}(k-1)) / t_{s}=\theta u(k-1)
$$

so, $\quad \dot{\hat{y}}(k)=\dot{\hat{y}}(k-1)+t_{s} \theta u(k-1)$

Relate Exp.(11),

$\dot{\hat{y}}(k)=\dot{\hat{y}}(k-1)+t_{s} \ddot{\hat{y}}(k-1)+\beta t_{s}(z(k-1)-\hat{y}(k-1))$, we have

$$
\dot{\hat{y}}(k)=\dot{\hat{y}}(k-1)+t_{s} \ddot{\hat{y}}(k-1)+\beta t_{s}(z(k-1)-\hat{y}(k-1))+t_{s} \theta u(k-1)
$$

Exp.(11) becomes:

$$
\left\{\begin{array}{l}
\hat{y}(k)=\hat{y}(k-1)+t_{s} \dot{\hat{y}}(k-1)+0.5 t_{s}^{2} \ddot{\hat{y}}(k-1)+\alpha t_{s}(z(k)-\hat{y}(k-1)) \\
\dot{\hat{y}}(k)=\dot{\hat{y}}(k-1)+t_{s} \ddot{\hat{y}}(k-1)+\beta t_{s}(z(k)-\hat{y}(k-1))+t_{s} \theta u(k-1) \\
\ddot{\hat{y}}(k)=\ddot{\hat{y}}(k-1)+\gamma t_{s}(z(k)-\hat{y}(k-1))
\end{array}\right.
$$

Exp.(12) is a time discrete observer with control input $u$ for the control system (3)

Using the transformation

$$
I+A t+0.5 A^{2} t^{2}=\phi
$$

We have the following time continuous observer of an unknown controlled plant

$$
\left\{\begin{array}{l}
\dot{\hat{y}}_{1}=\hat{y}_{2}+\alpha(z-\hat{y}) \\
\dot{\hat{y}}_{2}=\hat{y}_{3}+\beta(z-\hat{y})+\theta u \\
\dot{\hat{y}}_{3}=\gamma(z-\hat{y})
\end{array}\right.
$$

where $\hat{y}_{1}=\hat{y} ; \quad \hat{y}_{2}=\dot{\hat{y}} ; \quad \hat{y}_{3}=\ddot{\hat{y}}, \quad t_{s}$ is sample(/control) period, $u$ is control input to the plant system, $l \leq \theta \leq h, . l$ and $h$ are real boundary numbers.

Exp. (13) is the time continuous observer of an unknown controlled plant with control input $u$ for the control system (3), Exp. (13) is simply called OCICD(Observer with Control Input based on Classical Dynamics).

Lemma. Discrete systems (9) and (10) are completely controllable and observable.

Proof. The state matrix $\phi$ is time-invariant, as $t_{s}$ is determined in systems (9), we calculate

$$
\begin{gathered}
(\phi, \Gamma)=\left[\Gamma|\phi \Gamma| \phi^{2} \Gamma\right]=\left[\begin{array}{ccc}
0 & t_{s}^{2} / 2 & 2 t_{s}^{2} \\
0 & t_{s} & 2 t_{s} \\
1 & 1 & 1
\end{array}\right] \\
(\phi, c)=\left[c^{T}\left|\phi^{T} c^{T}\right|\left(\phi^{T}\right)^{2} c^{T}\right]=\left[\begin{array}{ccc}
1 & 1 & 1 \\
0 & t_{s} & 2 t_{s} \\
0 & t_{s}^{2} / 2 & 2 t_{s}^{2}
\end{array}\right]
\end{gathered}
$$

$\operatorname{rank}(\phi, \Gamma)=3, \operatorname{rank}(\phi, c)=3$; so discrete systems (9) and (10) are completely controllable and observable.

Theorem 1. Time continuous observer system (13) is consistent asymptotic stable.

Proof. We have proved discrete systems (9) and (10) are completely controllable and observable, time discrete observer (12) is obtained by Kalman filter, so time discrete observer (12) is consistent asymptotic stable when $\alpha, \beta$ and $\gamma$ are rightly selected. Time continuous observer (13) is 
obtained by transforming time discrete observer (12), so time continuous observer (13) is also consistent asymptotic stable, when $\alpha, \beta$ and $\gamma$ are rightly selected. From characteristic equation $|s I-A|=s^{3}+\alpha s^{2}+\beta S+\gamma$ of time continuous observer (13), if

$$
\alpha>0, \beta>0, \gamma>0 \text { and } \alpha \beta>\gamma,
$$

then time continuous observer system (13) is consistent asymptotic stable, based on Routh and Hurwitz's stability criterion.

We set

$$
\alpha=1 / t_{s}, \beta=0.5 / t_{s}^{2}, \gamma=0.088 / t_{s}^{3}
$$

in time continuous observer system (13) based on Exp. (14) and our experience.

Exp. (15) is only related to a sample (/control) period $t_{s}$, and independents from a controlled plant.

Theorem 2. $\hat{y}_{3}$ in time continuous observer system (13) is unbiased estimate value to $f\left(x_{1}, x_{2}, v(t)\right)$ in a second order state space model (3) :

Proof. It is known, Kalman filter is an unbiased estimator, time discrete observer (12) and time continuous observer system (13) are the special applications of Kalman filter, and second system (3) is completely controllable and observable, so time discrete observer (12) and time continuous observer system (13) are unbiased estimators to system (3).

Let

$$
x_{3}=f\left(x_{1}, x_{2}, v(t)\right)
$$

System (3) is written as:

$$
\left\{\begin{array}{l}
\dot{x}_{1}=x_{2} \\
\dot{x}_{2}=x_{3}+b u \\
\dot{x}_{3}=\dot{f}\left(x_{1}, x_{2}, v(t)\right) \\
y=x_{1}
\end{array}\right.
$$

Comparing Exp. (17) with Exp. (13):

$$
\left\{\begin{array}{l}
\dot{\hat{y}}_{1}=\hat{y}_{2}+\alpha(z-\hat{y}) \\
\dot{\hat{y}}_{2}=\hat{y}_{3}+\beta(z-\hat{y})+\theta u \\
\dot{\hat{y}}_{3}=\gamma(z-\hat{y}) \\
\hat{y}=\hat{y}_{1}
\end{array}\right.
$$

Because time continuous observer system (13) is unbiased estimator to system (3), we have:

$$
\hat{y}_{1} \rightarrow x_{1}=y, \hat{y}_{2} \rightarrow x_{2}, \hat{y}_{3} \rightarrow x_{3}=f\left(x_{1}, x_{2}, v(t)\right)
$$

We have proven $\hat{y}_{3}$ in time continuous observer system (13) is unbiased estimate value to $f\left(x_{1}, x_{2}, v(t)\right)$ in a second system (16).

We have the following observer system for a controlled plant process with acceleration $a=0$ :

$$
\begin{gathered}
\dot{\hat{y}}_{1}=\hat{y}_{2}+\alpha(z-\hat{y})+\theta u ; \quad \dot{\hat{y}}_{2}=\beta(z-\hat{y}) \\
\hat{y}=\hat{y}_{1}
\end{gathered}
$$

where $\alpha=1 / t_{s}, \beta=0.5 / t_{s}^{2}$

We have the following observer system for a controlled plant process with varying acceleration $a$

$$
\begin{gathered}
{\left[\begin{array}{c}
\dot{\hat{y}}_{1} \\
\dot{\hat{y}}_{2} \\
\dot{\hat{y}}_{3} \\
\dot{\hat{y}}_{4}
\end{array}\right]=\left[\begin{array}{cccc}
-\alpha & 1 & 0 & 0 \\
-\beta & 0 & 1 & 0 \\
-\gamma & 0 & 0 & 1 \\
-\theta & 0 & 0 & 0
\end{array}\right]\left[\begin{array}{l}
\hat{y}_{1} \\
\hat{y}_{2} \\
\hat{y}_{3} \\
\hat{y}_{4}
\end{array}\right]+\left[\begin{array}{cc}
\alpha & 0 \\
\beta & 0 \\
\gamma & \lambda \\
\theta & 0
\end{array}\right]\left[\begin{array}{l}
z \\
u
\end{array}\right]} \\
\hat{y}=\hat{y}_{1}
\end{gathered}
$$

where $\alpha=1 / t_{s}, \beta=0.5 / t_{s}^{2} \gamma=0.088 / t_{s}^{3}, \theta=0.01 / t_{s}^{4}$.

\section{Control System Design with OCICD and Removing Uncertain}

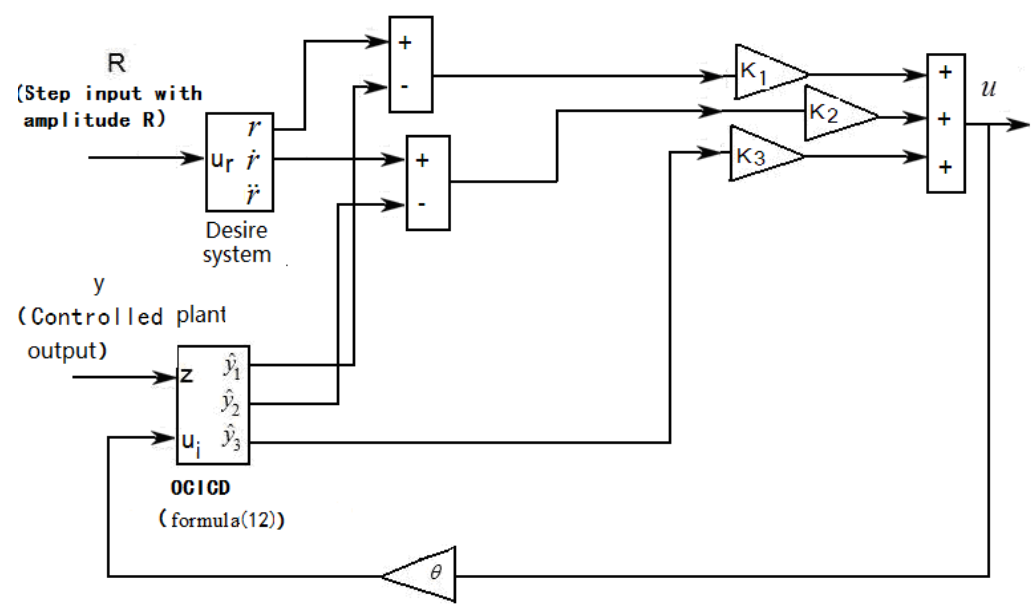


Fig 1. COCD (Controller with Observer based on Classical dynamics).

A controller and control system with OCICD are respectively designed shown in Fig.1 and Fig.3. The position, velocity and acceleration of control system output are negative feedback function in system. The controlled plant is a second order state space model (3), the second order system is of general model in process control system.

The controller of Fig. 1 is called as COCD (Controller with Observer based on Classical dynamics), it consists of setting desire system output locus, OCICD and controller output. The control system of Fig. 3 is called as CSCOCD (Control System with COCD).

\subsection{Setting Desire System Output Locus}

The desire system output $y$ is designed in COCD,

$$
y=R /\left(a s^{2}+b s+1\right)=G_{r}(s) R ;
$$

$$
G_{r}(s)=1 / /\left(a s^{2}+b s+1\right)
$$

where $R$ is the amplitude of step input,

$$
a=1 / w_{n}^{2}, b=2 \varsigma / w_{n}
$$

$w_{n}$ is the undamped natural frequency, $\zeta$ is the damping coefficient.

The desire transient process time $T$ is defined as the time in which $y$ approaches to $0.98 * R$ for the closed loop system, and $T$ is related to Exp. (22), (23) and (24).

$$
\varsigma=1, w_{n}=7 / T,
$$

then the desire system is determined, it is configured in state space model shown in Fig, $2, f_{1}=1 / a, f_{2}=b / a$ in Fig,2.

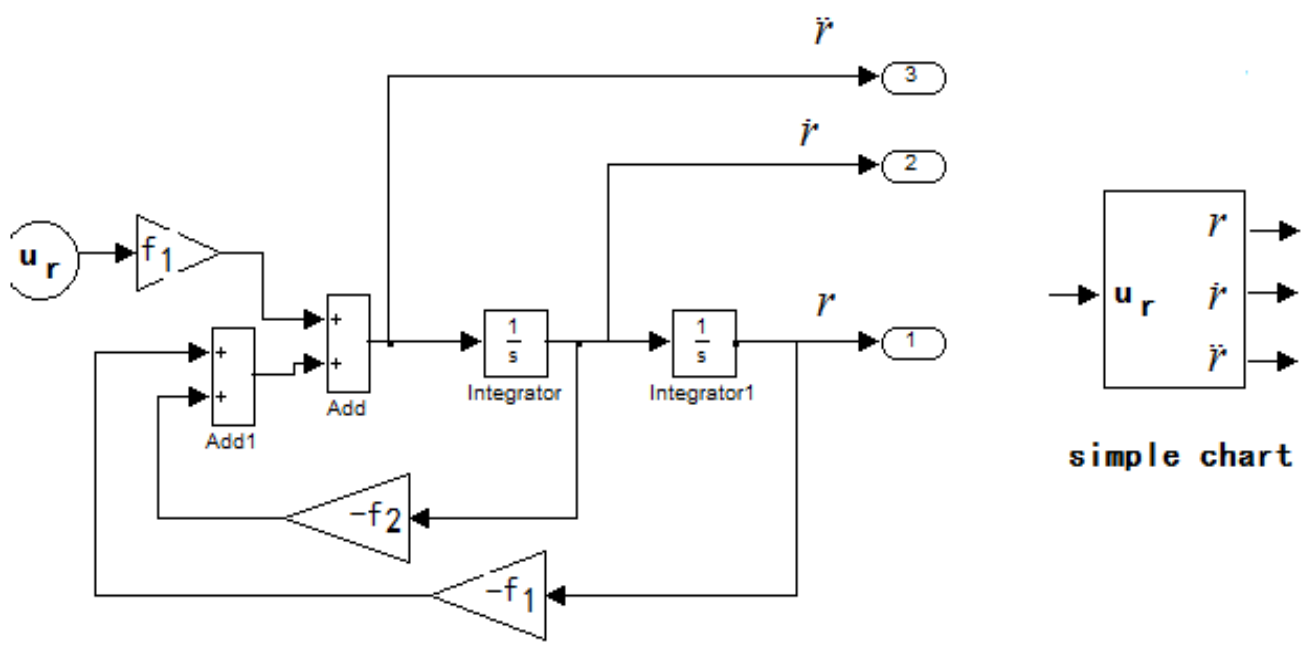

Fig 2. Desire system.

\subsection{Determining Parameters of OCICD}

The sample $t_{s}$ is determined by the sample theory and our experience:

$$
t_{s}=T / n, \quad n \in\left[\begin{array}{ll}
1000 & 2000
\end{array}\right]
$$

where $T$ is the desire transient process time, the $\alpha, \beta$ and $\gamma$ are calculated by Exp. (15).

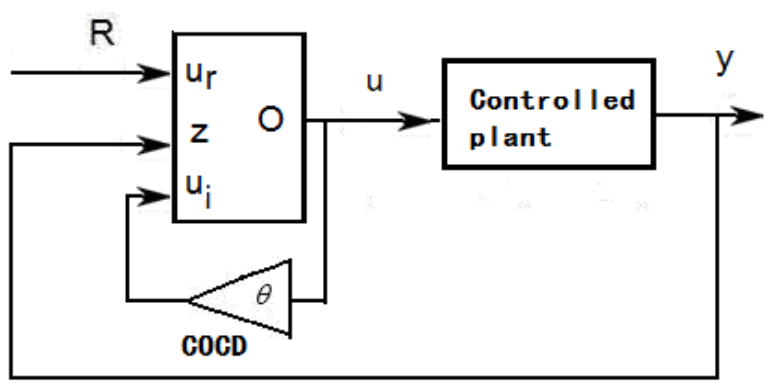

Fig 3. $C S C O C D($ Control system with $C O C D)$.

\subsection{Controller Output}

If the value $b$ in controlled plant state space model (17) is known, let

$$
u=\left(u_{0}-f\left(x_{1}, x_{2}, v(t)\right)\right) / b
$$

then controlled plant state space model (16) is changed to

$$
\left\{\begin{array}{l}
\dot{x}_{1}=x_{2} \\
\dot{x}_{2}=u_{0} \\
y=x_{1}
\end{array}\right.
$$

A suitable $u_{0}$ is selected, the transform function $G_{a}(s)$ between $y$ and $u_{0}$ 


$$
G_{a}(s)=[1,0]\left[\left[\begin{array}{ll}
s & 0 \\
0 & s
\end{array}\right]-\left[\begin{array}{ll}
0 & 1 \\
0 & 0
\end{array}\right]\right]^{-1}\left[\begin{array}{l}
0 \\
1
\end{array}\right]=1 / s^{2},
$$

If $f\left(x_{1}, x_{2}, v(t)\right)$ in controlled plant state space model (3) is uncertain, then the uncertain $f\left(x_{1}, x_{2}, v(t)\right)$ including unknown disturbance $v(t)$ are completely removed. We have proven that $\hat{y}_{3}$ in time continuous observer system (13) is unbiased estimate value to $f\left(x_{1}, x_{2}, v(t)\right)$ in Theorem 2 , so we select $K_{3}=1 / b$, and

$$
u=K_{1}\left(r-\hat{y}_{1}\right)+K_{2}\left(\dot{r}-\hat{y}_{2}\right)-K_{3} \hat{y}_{3}=u_{0}-\hat{y}_{3} / b
$$

where $u_{0}=K_{1}\left(r-\hat{y}_{1}\right)+K_{2}\left(\dot{r}-\hat{y}_{2}\right)$.

If controlled plant space model (16) is known, then

$$
\theta=b, K_{3}=1 / \theta,
$$

and $K_{1}$ and $K_{2}$ can be obtained by the solution of optimal linear-quadratic Gaussian algorithm(LQG) for the controlled plant space model (3).

If the plant state space model (17) is unknown, assuming

$$
l \leq b \leq h,
$$

we set firstly:

$$
\theta=(l+h) / 2, \quad K_{3}=1 / \theta, K_{1} \in\left[\begin{array}{ll}
1 & 1.5
\end{array}\right], \quad K_{2} \in\left[\begin{array}{ll}
0.5 & 2
\end{array}\right]
$$

then we can determine $\theta, K_{1}, K_{2}, K_{3}$ by several times tests based on the golden section.

It is important to determine desire transient process time $T$ without the mathematics model of controlled plant . Control engineers must estimate the motion (/response) velocity and completing time of controlled plant process based on analysis and understanding for a controlled plant process, just as the time program to plan a trip, we must estimate velocity and completing time of a vehicle.

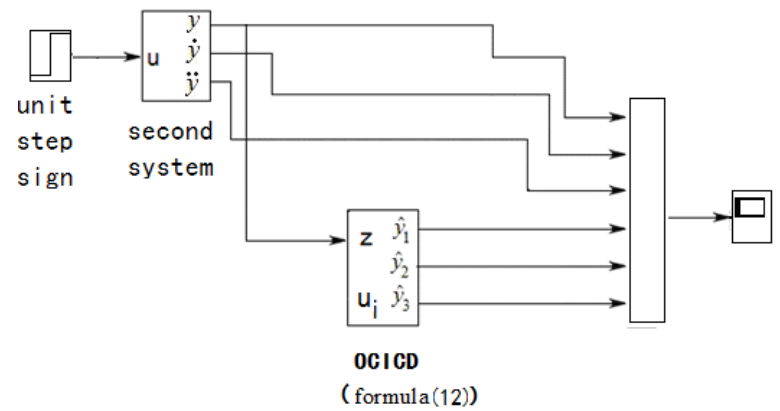

Fig 4. Inspecting the OCICD efficiency.

\section{Simulation and Application Examples with COCD}

\subsection{Simulation Example}

Fig.4 is simulated with Matlab to inspect the OCICD efficiency. The second system transfer function $\mathrm{G}_{p}(s)=1 /\left(0.21 s^{2}+0.37 s+1\right)$ is configured as Fig.2, where $f_{1}=4.76, f_{2}=1.76, \mathrm{R}=1, . \quad \mathrm{T}=1(\mathrm{sec}), \mathrm{t}_{s}=0.001, \alpha=1000$, $\beta=500000, \gamma=88000000$ in OCICD.

The simulated curves of OCICD outputs and the second system outputs are shown in Fig.5, the OCICD outputs are very close to the second system outputs, Fig.5 confirms OCICD (13) which is an unbiased estimator to a system.
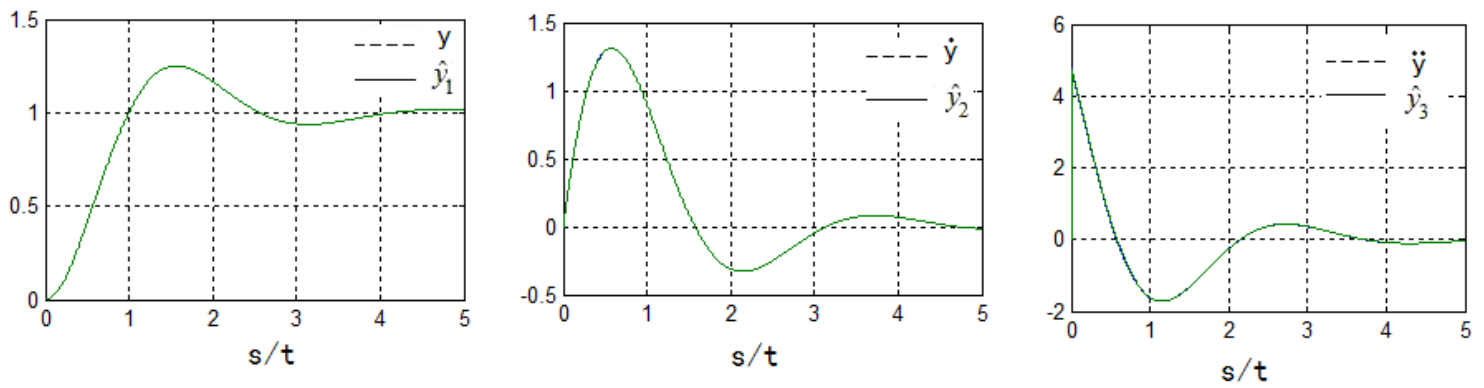

Fig 5. Comparing outputs of OCICD and the second system.

\subsection{Application Example}

CSCOCD of Fig. 3 is used to control the time-varying controlled plant with the transfer function $\mathrm{G}_{p 1}(s)=0.8 /\left(0.21 s^{2}+0.37 s+1\right) \quad$ and $\quad \mathrm{G}_{p 2}(s)=1.3 /\left(0.06 s^{2}+0.17 s+1\right)$ in some steel factory. The same parameters are selected for the time-varying controlled plants $\mathrm{G}_{p 1}(s)$ and $\mathrm{G}_{p 2}(s)$ :

$$
\mathrm{T}=1(\mathrm{sec}), \mathrm{t}_{s}=0.001, \alpha=1000, \beta=500000, \gamma=88000000
$$

in OCICD; $\mathrm{R}=1, f_{1}=45, \mathrm{f}_{2}=13.5$ in the desire system of Fig. $2 ; K_{1}=1, K_{2}=1.5, K_{3}=0.007, \theta=145$. 

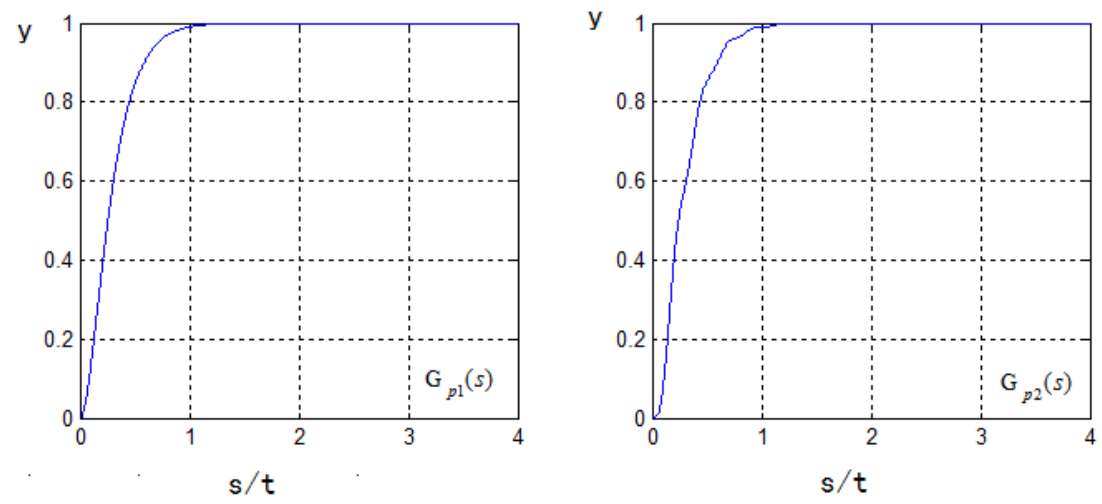

Fig 6. Outputs of the closed loop system of $\mathrm{G}_{p 1}(s)$ and $\mathrm{G}_{p 2}(s)$.

The closed loop system outputs of $\mathrm{G}_{p 1}(s)$ and $\mathrm{G}_{p 2}(s)$ with the same control parameters are shown in Fig.6, and Fig.6. confirms good robust performance of CSCOCD.

\section{Conclusion}

(1) The paper designs the control system based on classical dynamics and explains classical dynamics philosophy of the control system design.

(2) The control system is of good robust performance, it can overcome the uncertain of a controlled plant and remove disturbances into the system in the paper.

(3) The design of control system needs not an exact mathematics model of the controlled plant. If we can estimate the values $T$ in Exp. (24) and $l, h$ in Exp. (29), we can design the control system without an exact mathematics model of the controlled plant.

\section{References}

[1] Kalman, R. E., 'On the general theory of control systems', Ire Transactions On Automatic Control (1959) Volume: 4, Issue: 3, Publisher: Butterworth, London, pp. 110-110.

[2] Kudva, P., Viswanadham, N., Ramakrishna, A., 'Observers for linear systems with unknown inputs', IEEE Trans. Automat. Control, 1980. pp.113 115.

[3] [3] R. E. Kalman, R. S. Bucy, 'New results in linear filtering and prediction theory', J. Basic Eng, 1961 [7]. pp.111-145.

[4] Corless. M, Tu.J State/input estimation for a class of uncertain system system,Automatica, 1998, 34(6); pp.757-764.

[5] Jingqing Han, 'Nonlinear State Error Feedback Control', Control and Decision (Chinese), Vol.10, No.3, Nov., 1994, pp.221-225.

[6] Kai Pingan etc., 'Advanced control technology in fire power plant', (Chinese), China Electric Power Publishing House, 4, 2010. pp.65-76. 